\title{
TESTS FOR SUBSTRUCTURE IN GRAVITATIONAL LENSES
}

\author{
C. S. KochaneK ${ }^{1}$ and N. Dalal ${ }^{2,3}$ \\ Received 2003 February 3; accepted 2004 March 25
}

\begin{abstract}
The flux anomalies in four-image gravitational lenses can be interpreted as evidence for the dark matter substructure predicted by cold dark matter (CDM) halo models. In principle, these flux anomalies could arise from alternate sources, such as absorption, scattering, or scintillation by the interstellar medium (ISM) of the lens galaxy, problems in the ellipsoidal macro models used to fit lens systems, or stellar microlensing. We apply several tests to the data that appear to rule out these alternate explanations. First, the radio flux anomalies show no significant dependence on wavelength, as would be expected for almost any propagation effect in the ISM or microlensing by the stars. Second, the flux anomaly distributions show the characteristic demagnifications of the brightest saddle point relative to the other images expected for low optical depth substructure, which cannot be mimicked by either the ISM or problems in the macro models. Microlensing by stars also cannot reproduce the suppression of the bright saddle points if the radio source sizes are consistent with the Compton limit for their angular sizes. Third, while it is possible to change the smooth lens models to fit the flux anomalies in some systems, we can rule out the necessary changes in all systems where we have additional lens constraints to check the models. Moreover, the parameters of these models are inconsistent with our present observations and expectations for the structure of galaxies. We conclude that low-mass halos remain the best explanation of the phenomenon.
\end{abstract}

Subject headings: dark matter — galaxies: halos — gravitational lensing

\section{INTRODUCTION}

In Dalal \& Kochanek (2002, hereafter DK02), we demonstrated that the incidence of anomalous flux ratios in gravitational lenses was consistent with the expected mass fraction of satellites found in cold dark matter (CDM) halo simulations (e.g., Kauffmann et al. 1993; Moore et al. 1999; Klypin et al. 1999; Bode et al. 2001; Zentner \& Bullock 2003). Our quantitative estimate for the substructure mass fraction was part of an outburst of interest in CDM substructure and anomalous flux ratios in individual gravitational lenses (e.g., Mao \& Schneider 1998; Keeton 2001a; Bradac et al. 2002; Chiba 2002; Metcalf 2002) and in CDM models (e.g., Metcalf \& Madau 2001; Chiba 2002; Zentner \& Bullock 2003), as well as the difficulty in explaining the anomalous flux ratios of gravitational lenses based on the properties of the primary lens galaxy (e.g., Metcalf \& Zhao 2002; Keeton et al. 2003; Evans \& Witt 2003; Möller et al. 2003; Quadri et al. 2003). In DK02 we argued that alternate explanations to substructure for the anomalous flux ratios were unlikely, but we did not explore the alternate possibilities in detail or develop tests to distinguish substructure from the alternatives. We do so in the current paper.

The alternatives to substructure fall into three broad categories: propagation effects in the interstellar medium (ISM) of the lens galaxy, problems in the "macro" models for the gravitational potential of the lens galaxy, and confusing "microlensing" by the stars in the lens galaxy with the effects of more massive satellites. In this paper we consider all three of these possibilities. In $\S 2$, we study the wavelength dependence of the flux anomalies and develop a simple statistical test to demonstrate that the anomalous flux ratio problem

\footnotetext{
${ }^{1}$ Department of Astronomy, Ohio State University, 140 West 18th Avenue, Columbus, OH 43210; ckochanek@astronomy.ohio-state.edu.

${ }_{2}$ School of Natural Sciences, Institute for Advanced Study, Einstein Drive, Princeton, NJ 08540; neal@ias.edu.

${ }^{3}$ Hubble Fellow.
}

must be due to gravity. In $\S 3$, we show that deviations from elliptical shapes appear unable to account for flux anomalies and that errors in the model for the potential of the primary lens have statistical properties differing from those created by substructure and observed in the data. In $\S 4$, we show that it is difficult to explain the anomalous flux ratios of the radio lenses using microlensing. We summarize our results and outline further tests in $\S 5$.

\section{RULING OUT THE ISM}

The ISM can affect flux ratios through either absorption or scattering. In optical/IR observations, many gravitational lenses show wavelength-dependent flux ratios consistent with the effects of dust extinction (e.g., Falco et al. 1999). In the radio, some lenses show the effects of scatter broadening by electrons at low frequencies (e.g., B1933+503; Marlow et al. 1999a), although in none of these systems is there evidence for a net change in the radio flux. In this section, we examine whether the radio lenses show any frequency-dependent changes in their flux ratios that could be a signature of the ISM modifying the observed fluxes and develop a simple, nonparametric test to distinguish the effects of the ISM from the effects of gravity.

\subsection{The Frequency Dependence of Anomalous Flux Ratios}

Almost all mechanisms by which the ISM can modify flux ratios should show frequency-dependent effects, since most scattering processes relevant to the fluxes of radio images become weaker at higher frequencies. For example, weak scintillation causes flux perturbations with a scaling (assuming Kolmogorov turbulence) of $\propto \nu^{-17 / 12}$ (Narayan 1992), and the optical depth for free-free absorption scales roughly as $\propto \nu^{-2.1}$ (e.g., Mezger \& Henderson 1967). Thus, one way to constrain the ISM as an explanation of the anomalous flux ratios is to examine the allowed frequency dependence of the 
effect. If the source has an intrinsic spectrum $f_{\nu, s}$ as a function of frequency $\nu$, then in the absence of any perturbations from the ISM, the images have a spectrum $f_{\nu, i}=\left|M_{i}\right| f_{\nu, s}$, given the (signed) image magnification $M_{i}$. If the ISM modifies the fluxes through a frequency-dependent optical depth $\tau_{\nu, i}$, then the image fluxes become $f_{\nu, i}=\left|M_{i}\right| f_{\nu, s} \exp \left(-\tau_{\nu, i}\right)$.

We examined the optical depth function using the four- (or more) image radio lenses with fluxes measured at $5 \mathrm{GHz}$ and either 8 or $15 \mathrm{GHz}$ (MG 0414+0534: Katz et al. 1997; B0712+472: Jackson et al. 1998; B1359+154: Myers et al. 1999; B1422+231: Patnaik \& Narasimha 2001; B1555+375: Marlow et al. 1999b; B1608+656: C. D. Fassnacht 2003, private communication; B1933+503: Sykes et al. 1998; and B2045+265: Fassnacht et al. 1999). We used the same models and magnification estimates as were used to estimate the substructure fraction, with a 5\% lower bound on the flux errors. The simplest way to illustrate the problem is to assume that one image is strongly affected by the ISM while the others suffer from little or no absorption or scattering. For each image in a lens, we used the fluxes of the other three to estimate the intrinsic spectrum of the source. From this estimate of the intrinsic spectrum we computed the optical depth needed to reproduce the observed flux of the remaining image, by computing the necessary optical depth at $5 \mathrm{GHz}, \tau_{5}$, and its spectral index, $\alpha$, under the assumption that $\tau \propto \nu^{\alpha}$ between $5 \mathrm{GHz}$ and the available higher frequency. We estimated the errors solely from the published measurement errors, so they include no uncertainties arising from the lens model or any time variability between the measurement epochs for the different frequencies.

The results are shown in Figure 1. We have coded the images by their parities (minima vs. saddle points) and relative fluxes (brightest vs. faintest for each parity), because we show below that the distributions of the model flux residuals depend strongly on these image identifications. This is seen in Figure 1 as the concentration of the bright saddle point images in the direction of higher optical depth compared to the other images. We have dropped four (of 32) images in which the estimated optical depth changes sign between the higher and lower frequencies and it is impossible to define a spectral index. Except at zero optical depth, where the slope estimates become unstable and have larger uncertainties, the lenses broadly require $\alpha \simeq 0$. Since most radio propagation effects have strong wavelength scalings, the ISM seems an unlikely explanation.

\subsection{A Statistical Test for Substructure}

While the spectral indices required to explain the anomalous flux ratios are peculiar for standard scattering or absorption processes, we would prefer to have a test that can distinguish any ISM effect from the gravitational effects of substructure. One approach is to note that the physical properties of the ISM are a local property of the lens and should have no knowledge of the global properties of the lens geometry. Radial gradients in the properties of the ISM should matter little, because in each lens the images of interest effectively lie at the same radius and there is no simple angular correlation between the positions of the images and the major axis of the galaxy. Moreover, creating the anomalous flux ratios depends on the presence of clumped ISM components rather than smoothly distributed components, both to create physical conditions extreme enough to have an effect and to differentially affect the lensed images. The only exception to this rule arises for effects such as scintillation or scatter

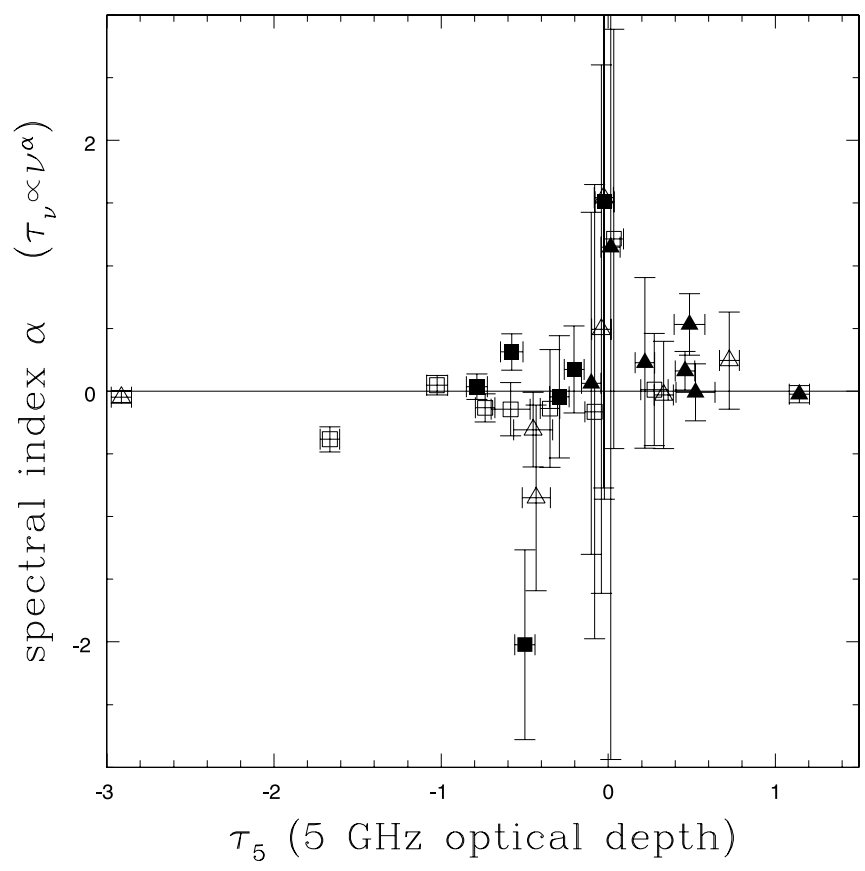

FIG. 1.-Estimates of the ISM properties needed to explain the anomalous flux ratios. We show an estimate of optical depth at $5 \mathrm{GHz}, \tau_{5}$, and the spectral index $\alpha$, where $\tau \propto \nu^{\alpha}$, needed to bring the observed flux of each image into agreement with the average source properties predicted by the other three images. The images are coded by their parities and relative fluxes, with squares used for minima (positive parity) and triangles for saddle points (negative parity). Filled symbols are used for the brightest image of each parity and open symbols for the faintest image. Note the concentration of the brightest saddle points (minima) toward positive (negative) optical depth.

broadening, in which the effect diminishes as the source size becomes larger-for these cases, the ISM should preferentially perturb the least magnified images, because they are the most compact.

The effects of gravity, however, are not determined purely by local conditions, because the image positions and fluxes are determined by the competition between the local gravitational potential and the geometric time delay. The magnification tensor, whose eigenvalues determine the image parities, depends on the projected surface density, the projected tidal shear in the gravity, and the redshifts of the lens and the source. As a result, we can attach a global identification to each image based on its parity (maximum, minimum, and saddle point of the time delay surface) as well as its magnification, with which the ISM should show little (magnification) or no (parity) correlation. Image parities and magnification orderings (but not the precise magnifications) are generic predictions of successful lens models. In particular, for a fourimage lens (or "quad"), the images alternate parities in the sequence minimum-saddle point-minimum-saddle point as we go around the lens. We can also identify the most and least magnified image of the two minima or saddle points from the geometry of the lens. Unlike those of the ISM, the effects of substructure depend on the image parity and magnification. The fluxes of highly magnified images are more unstable to small gravitational perturbations than the fluxes of less magnified images (as originally emphasized by Mao \& Schneider 1998). Most importantly, the magnification perturbations created by low optical depth substructure have a very strong dependence on the image parity, because the perturbations to the fluxes of the saddle points are skewed in the direction of demagnification (Schechter \& Wambsganss 2002; Keeton 


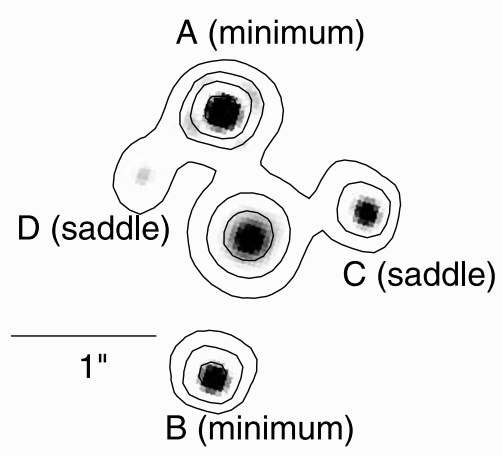

FIG. 2.-The most spectacular example of a suppressed saddle point, SDSS J0924+0219 (Inada et al. 2003). The D image should be comparable in brightness to the A image, but is actually an order of magnitude dimmer. The $\mathrm{A}$ and $\mathrm{B}$ images are minima, while $\mathrm{C}$ and $\mathrm{D}$ are saddle points. The contours are spaced by factors of 2 from the peak of the A image. The lens galaxy is seen at the center. In this infrared image, the suppression of the saddle point could be due to either microlensing or substructure.

2003). Figure 2 shows the most spectacular example of a suppressed saddle point, image $D$ of the four-image lens SDSS J0924+0219 (Inada et al. 2003). The D image, which should be comparable in brightness to the A image, is observed to be an order of magnitude fainter. Substructure in the gravitational potential, either satellites or stars, should make the flux statistics of the brightest saddle point (as predicted by smooth potentials) different from those of either the brightest minimum (A) or the two fainter images (the minimum $\mathrm{B}$ and the saddle point C; Metcalf \& Madau 2001; Keeton 2003; Schechter \& Wambsganss 2002; Bradac et al. 2002). This leads to a simple nonparametric test for distinguishing the effects of the ISM from the effects of gravity-if the flux anomalies depend on parity and magnification, they cannot be due to the ISM.

We first tested the effects of substructure on the distribution of flux residuals. We took the same sample of seven lenses we used in DK02 (MG 0414+0534: Hewitt et al. 1992; B0712+472: Jackson et al. 1998; PG 1115+080: Weymann et al. 1980; B1422+231: Patnaik et al. 1992; B1608+656: Fassnacht et al. 1996; B1933+503: Sykes et al. 1998; and B2045+265: Fassnacht et al. 1999). The data were fitted using standard macro models (singular isothermal ellipsoids [SIE] plus external shear) to generate an initial model. We then added a substructure mass fraction $f_{\text {sat }}$ near the images, modeling the substructure as tidally truncated isothermal spheres with critical radius $b=00^{\prime \prime} 001$ (corresponding to approximately $10^{6} M_{\odot}$ ). With the substructure added, we reidentified the lensed images and their fluxes and added astrometry $(0.003 \mathrm{rms})$ and flux $(10 \% \mathrm{rms})$ measurement errors to generate a model data set with substructure. These model data were then refitted using the standard macro models, including no weight on the fit to the fluxes of the images. We then compare the observed image fluxes, $f_{\mathrm{obs}, i}$ to the model image fluxes, $f_{\bmod , i}=\left|M_{i}\right| f_{\text {src }}$, found given the magnification $M_{i}$ predicted by the model fitted to the perturbed data. We generated 10 such realizations for each of the seven lenses. As expected, the residual distribution for the brightest saddle point is markedly different from that for all other

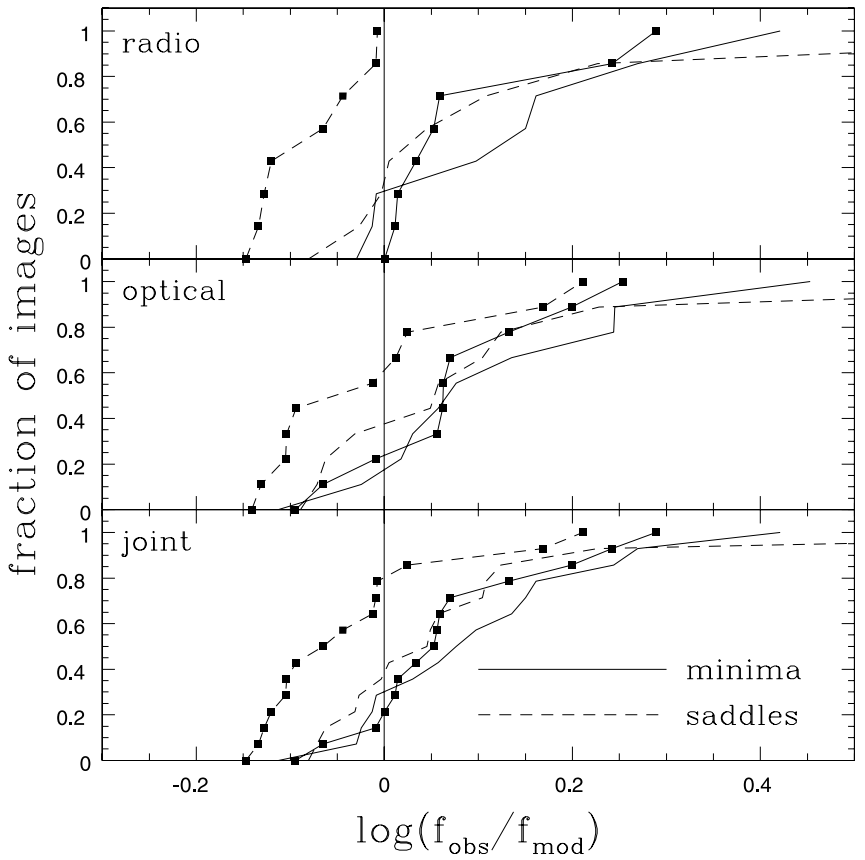

FIG. 3.-Cumulative distributions of model flux residuals, $\log \left(f_{\text {obs }} / f_{\bmod }\right)$, in the real data, assuming constant fractional flux errors for each image. The solid (dashed) lines are for minima (saddle points), with squares (no squares) for the distribution corresponding to the most (least) magnified image. From top to bottom, the distributions are shown for samples of eight radio, 10 optical, or 15 total four-image lenses. If the flux residuals were created by propagation effects, we would not expect the distributions to depend on the image parity or magnification, while if they were due to low optical depth substructure, we would expect the distribution for the brightest saddle points to be shifted to lower observed fluxes.

images unless the substructure fraction is so low as to be masked by the random flux errors.

We then repeated the test for the real data. The same standard macro models were used for each system, and we again assigned no weight to fitting the observed image fluxes. For radio lenses we used the highest frequency measurements from either the VLA ${ }^{4}$ or MERLIN, and for the optical lenses we used the flux ratios at the longest available wavelength (generally, the $H$ band, where none of the systems shows significant extinction). This gave us a sample of eight radio systems (adding B0128+437 [Phillips et al. 2000] and B1555+375 [Marlow et al. 1999b] to the DK02 sample), 10 optical systems, ${ }^{5}$ and 15 joint systems. For our standard model, we estimated the source flux using constant fractional errors on the image fluxes. Figure 3 shows the distribution for the residuals, $\log \left(f_{\text {obs }} / f_{\text {mod }}\right)$, for all currently available quads containing either compact radio sources or bright quasars. Several systems appear in both categories, and for these systems we use the radio fluxes for the joint sample. The distributions of the

\footnotetext{
4 The VLA (Very Large Array) is a facility of the National Radio Astronomy Observatory (NRAO). The NRAO is a facility of the National Science Foundation operated under cooperative agreement by Associated Universities, Inc.

5 The systems are the four-image quasar lenses HE 0230-2130 (Wisotzki et al. 1999), HE 0435-1223 (Wisotzki et al. 2002), HS 0810+2554 (Reimers et al. 2002), RX J0911+0551 (Bade et al. 1997), PG 1115+080 (Weymann et al. 1980), H1413+117 (Magain et al. 1988), Q2237+030 (Huchra et al. 1985), B1422+231 (Patnaik et al. 1992), B2045+265 (Fassnacht et al. 1999), and MG 0414+0534 (Hewitt et al. 1992). The latter three systems are also in the radio sample. We used the CASTLES $H$-band fluxes, if available, and otherwise the I-band fluxes.
} 
residuals differ for minima and saddles, in the sense that saddle points tend to be fainter than expected and minima tend to be brighter-just as expected for substructure.

We used the Kolmogorov-Smirnov (K-S) test to estimate the significance of the differences. If we simply compare the distributions of $\log \left(f_{\text {obs }} / f_{\text {mod }}\right)$ for minima and saddle points, we find that the K-S test probabilities for the two distributions to be the same are $2.3 \%, 28 \%$, and $5.5 \%$ for the radio, optical, and joint samples respectively. Theoretically, we expect the most highly magnified saddle point to have the most discrepant residual distribution, because the high magnification makes the flux unstable to perturbations and because the theoretical studies of low optical depth substructure predict that it should show the largest differences. The K-S test probabilities for the most magnified saddle point to have the same residual distribution as the other three images are only $0.04 \%, 5 \%$, and $0.3 \%$ for the radio, optical, and joint samples, respectively. In each case, the most highly magnified minimum shows the nextlowest probability for a residual distribution agreeing with the other images $(1.9 \%, 59 \%$, and $18 \%$ respectively), but the significance is lower. In the case of substructure, the magnification perturbation distribution for the minima is harder to differentiate from the measurement uncertainties, which probably dominated the residuals for the faintest images.

We can check these significance estimates by examining trials in which the identifications of the images are randomized. We made $10^{4}$ trials in which we assigned the image identifications (1: brightest minimum; 2 : faintest minimum; 3: brightest saddle point; 4: faintest saddle point) to the images at random, with the restriction that each lens always have the four possible image types. We then estimated the probability that these random assignments could produce results similar to that found for the true assignments. The fraction of the time the observed values of the K-S statistic were exceeded tracked the K-S test probability estimates well. For example, in only $2.3 \%, 18 \%$, and $4.8 \%$ of the trials did the K-S statistic for the comparison between the distributions of minima and saddle points exceed that of the true distribution. We also examined the distribution of the K-S statistic if we bootstrap-resampled the lens sample. The K-S statistics and probabilities were typical of any sample of lenses drawn (with replacement) from the available four-image lenses, so the results are not dependent on any particular system or small subset of systems.

We also examined weighting schemes for estimating the source flux other than assuming constant fractional errors for the observed image fluxes. Note, however, that our test is independent of any simple rescaling of the source flux. The K-S test makes a nonparametric comparison of the $\log \left(f_{\text {obs }} / f_{\text {mod }}\right)$ distributions, and a uniform change in the source flux simply shifts the distribution. If we assume constant flux errors rather than constant fractional errors, the probability that the saddles and minima have the same distribution is again $2.3 \%$. If we set the source flux using the fluxes of the faintest minimum and saddle, then the probability that the brightest minimum and saddle have the same residual distribution for the radio lenses is $0.02 \%$.

Finally, we can compare the observed distribution to either those expected for Gaussian errors or those we calculated for our Monte Carlo simulations of substructure. For example, while the overall residual distributions of the images are well fitted by a lognormal distribution of width $\sigma_{\ln f}=0.24$, the residual distributions of the images separately are grossly inconsistent with lognormal distributions. The K-S test likelihood for the joint distribution of all images matching a lognormal distribution is 0.26 , while the likelihood for the distributions of the individual types matching a single lognormal distribution is $1.5 \times 10^{-5}$. This is simply another way of quantifying the fact that the residual distributions depend on the image parities and flux orderings. If we compare the residual distributions by image for the radio lens sample to the distributions predicted in the Monte Carlo simulations, we find $\mathrm{K}-\mathrm{S}$ test probabilities for the distributions to be the same of $4 \times 10^{-7}, 2 \times 10^{-5}, 2 \times 10^{-4}, 0.02,0.3$, and 0.05 for the cases with satellite mass fractions of $f_{\text {sat }}=0.002,0.01,0.02,0.05$, 0.10 , and 0.20 , respectively. While we do not, at present, feel that this is as optimal a way of estimating $f_{\text {sat }}$ as the methods we used in DK02, it emphasizes the consistency of the observed dependence of the residual distribution on parity and flux with that expected for substructure.

\section{RULING OUT SYSTEMATIC PROBLEMS IN THE MODEL POTENTIALS}

If the anomalous flux ratios cannot be explained by propagation effects in the ISM, the only alternative to some form of substructure in the gravitational field is a systematic problem in the assumed macro model for the lens galaxy. Previous studies of the effects of changes in the macro model show that changes in the radial structure from the standard SIEs with external shear do not provide a solution (Metcalf \& Zhao 2002; Keeton et al. 2003). This makes physical sense, because the images in most of the systems with anomalous flux ratios all lie close to the Einstein radius of the lens, making it difficult for changes in the radial profile to produce changes in the flux ratios. We focus on changes in the angular structure because they can more easily shift the positions of the tangential critical line so as to change the image fluxes.

\subsection{Lens Models with Deviations from Ellipsoidal Structure}

We modified the gravitational potential by adding higher order multipoles of the form

$$
\phi_{m}=\frac{\epsilon_{m}}{m} r \cos m\left(\theta-\theta_{m}\right)
$$

to the primary lens galaxy. These correspond to a surface density

$$
\kappa_{m}=\frac{\epsilon_{m}}{r} \frac{1-m^{2}}{m} \cos m\left(\theta-\theta_{m}\right)
$$

and match the expansion used by Evans \& Witt (2003). The new terms have been included in a revised version of the lensmodel package (Keeton 2001b). ${ }^{6}$ In lensmodel, the surface density of the SIE model is defined by

$$
\kappa=\frac{1}{\sqrt{2}} \frac{b}{r} \frac{q}{\left[1+q^{2}-\left(1-q^{2}\right) \cos 2\left(\theta-\theta_{2}\right)\right]^{1 / 2}},
$$

where $q=1-\epsilon_{2}$ is the axis ratio and $\theta_{2}$ is the orientation of the major axis. The critical line, which is an isodensity contour with $\kappa=1 / 2$ for the SIE, has a major axis of $b$ and a minor axis of $b q$. Thus, if we add an $m=4$ term to an SIE model, these normalizations imply a standard amplitude for the deviation of an isophote from the ellipse of $a_{m}=\epsilon_{m}(1-$ $\left.m^{2}\right) / m b$. Positive $\epsilon_{4}$ corresponds to positive $a_{4}$ and "disky"

\footnotetext{
${ }^{6}$ The lensmodel package now allows terms of the form $\left(\epsilon_{m} / m\right)$ $r^{n} \cos m\left(\theta-\theta_{m}\right)$, where the normalization was chosen to reduce to an external shear for $n=2$ and $m=2$.
} 
TABLE 1

Basic Model Fits

\begin{tabular}{|c|c|c|c|c|c|c|}
\hline Lens & Case & $N_{\text {dof }}$ & $\chi_{\mathrm{tot}}^{2}-\chi_{\mathrm{flx}}^{2}$ & $\chi_{\mathrm{flx}}^{2}$ & $\left|a_{3}\right|$ & $\left|a_{4}\right|$ \\
\hline \multirow[t]{4}{*}{ MG $0414+0534 \ldots \ldots \ldots$} & Astrometry & 2 & 12.8 & 41.2 & $\ldots$ & $\ldots$ \\
\hline & Astrometry+flux & 5 & 10.0 & 35.5 & $\ldots$ & $\ldots$ \\
\hline & $\delta \theta_{m} \equiv 0$ & 3 & 2.4 & 19.1 & 0.011 & 0.020 \\
\hline & Full & 1 & 6.3 & 14.4 & 0.005 & 0.040 \\
\hline \multirow[t]{4}{*}{ B0712+472.. } & Astrometry & 1 & 4.2 & 112.7 & $\ldots$ & .. \\
\hline & Astrometry+flux & 4 & 31.8 & 15.5 & $\ldots$ & . \\
\hline & $\delta \theta_{m} \equiv 0$ & 2 & 6.6 & 21.4 & 0.095 & 0.178 \\
\hline & Full & 0 & 1.9 & 17.2 & 0.242 & 0.416 \\
\hline \multirow[t]{4}{*}{ PG $1115+080 \ldots \ldots \ldots \ldots$} & Astrometry & 2 & 2.9 & 57.0 & $\ldots$ & $\ldots$ \\
\hline & Astrometry+flux & 5 & 10.0 & 40.3 & $\ldots$ & \\
\hline & $\delta \theta_{m} \equiv 0$ & 3 & 2.2 & 18.3 & 0.006 & 0.022 \\
\hline & Full & 1 & 2.9 & 3.7 & 0.022 & 0.051 \\
\hline \multirow[t]{4}{*}{ B1422+231................... } & Astrometry & 1 & 2.1 & 12.3 & $\ldots$ & . \\
\hline & Astrometry+flux & 4 & 2.7 & 11.2 & $\ldots$ & $\ldots$ \\
\hline & $\delta \theta_{m} \equiv 0$ & 2 & 2.7 & 11.3 & 0.000 & 0.000 \\
\hline & Full & 0 & 0.5 & 0.1 & 0.089 & 0.069 \\
\hline \multirow[t]{4}{*}{$\mathrm{B} 1608+656 \ldots \ldots \ldots \ldots \ldots$} & Astrometry & 2 & 1.8 & 3.9 & $\ldots$ & $\ldots$ \\
\hline & Astrometry+flux & 5 & 1.6 & 0.7 & $\ldots$ & .. \\
\hline & $\delta \theta_{m} \equiv 0$ & 3 & 2.2 & 0.5 & 0.000 & 0.000 \\
\hline & Full & 1 & 4.1 & 0.6 & 0.000 & 0.000 \\
\hline \multirow{4}{*}{$\mathrm{B} 1933+503 \ldots \ldots \ldots \ldots$} & Astrometry & 1 & 0.5 & 254.2 & $\ldots$ & $\ldots$ \\
\hline & Astrometry+flux & 4 & 19.2 & 137.4 & $\ldots$ & $\ldots$ \\
\hline & $\delta \theta_{m} \equiv 0$ & 2 & 1.6 & 0.1 & 0.011 & 0.137 \\
\hline & Full & 0 & 1.3 & 0.0 & 0.079 & 0.138 \\
\hline \multirow[t]{4}{*}{ B2045+265 $\ldots \ldots \ldots \ldots \ldots$} & Astrometry & 1 & 1.4 & 302.0 & $\ldots$ & $\ldots$ \\
\hline & Astrometry+flux & 4 & 2.1 & 301.4 & $\ldots$ & $\ldots$ \\
\hline & $\delta \theta_{m} \equiv 0$ & 2 & 2.2 & 301.4 & 0.000 & 0.000 \\
\hline & Full & 0 & 1.9 & 301.3 & 0.001 & 0.000 \\
\hline
\end{tabular}

NoTES.-Four model cases are fitted: astrometry and astrometry+flux fit standard models (SIE+shear) to the image positions and fluxes. The $\delta \theta_{m} \equiv 0$ case adds the $m=3$ and 4 multipoles constrained to be aligned with the ellipsoid $\left(\theta_{m}=\theta_{2}\right)$, and the "full" models allow any orientation. We show the number of degrees of freedom $N_{\mathrm{dof}}$, the goodness of fit $\chi_{\mathrm{tot}}^{2}-\chi_{\mathrm{fl}}^{2}$ for the constraints other than the flux ratios, and the goodness of fit to the fluxes $\chi_{\mathrm{fl}}^{2}$, assuming $5 \%$ errors in the fluxes. The amplitudes of the multipoles are given by $\left|a_{3}\right|$ and $\left|a_{4}\right|$. Very weak priors are included on the ellipticity and shear.

density contours when the $m=4$ component is aligned with the quadrupole $\left(\theta_{4}=\theta_{2}\right)$. Galaxies with "boxy" isophotes will have negative $a_{4}$ and $\epsilon_{4}$.

We restrict our attention to adding $m=3$ and 4 terms to the potential of the primary lens galaxy. This should be sufficient to indicate their potential importance without the models being too underconstrained. Observational evidence indicates that the amplitude of these higher order multipoles is small. For example, Bender et al. (1989) and Rest et al. (2001) examine a sample of local ellipticals and quantify the deviations of the isophotes from ellipses. They find that the deviations are dominated by the $m=4$ term, $\propto a_{4} \cos \left(\theta-\theta_{4}\right)$, with a typical amplitude of $\left|a_{4}\right| \sim 0.01$. Deviations from ellipticity in projections of simulated halos are of comparable amplitude (Heyl et al. 1994; Burkert \& Naab 2003). Considerably less information is available on the distribution of misalignment angles, $\theta_{m}-\theta_{2}$, between the higher order multipoles and the dominant ellipse. We know from the statistics of lens models that the major axes of the mass and the light are closely aligned (Kochanek 2002). The effects of these higher order terms on lenses were first studied by Evans \& Witt (2001), who pointed out that larger values of $a_{4}$ than are typically observed would lead to significant cross sections for lenses producing more than four observable images.

We fitted the seven lenses in the DK02 sample with a sequence of four models whose results are presented in Table 1. In the first model ("astrometry") we fitted only the image positions, using our standard SIE plus external shear model. In the second model ("astrometry+flux") we added the constraints from the image fluxes, assuming 5\% uncertainties. In either case, six of the seven lenses have unacceptably high $\chi_{\mathrm{flx}}^{2}$ fit statistics for the image fluxes, even though the standard models provide good fits to the astrometric data, $\chi_{\mathrm{tot}}^{2}-\chi_{\mathrm{fx}}^{2}$. Only B1608+656 has a $\chi_{\mathrm{flx}}^{2}$ largely consistent with $5 \%$ measurement uncertainties. The failure of these models to reproduce the flux ratios is the origin of the entire problem of flux ratio anomalies. In the third model we added the $m=3$ and 4 multipoles to the potential of the primary lens, but constrained them to be aligned with the ellipsoid $\left(\theta_{m}=\theta_{2}\right.$, but allowing any sign for $\epsilon_{m}$ ). For MG 0414+0534, B0712+472, PG $1115+080$, and $\mathrm{B} 1933+503$, the new terms lead to a significant reduction in $\chi_{\mathrm{ffx}}^{2}$, but in no case does it become a statistically acceptable fit. The amplitudes of the multipoles are unreasonably large for B0712+472 and B1933+503. Finally, in the fourth model, we allowed the $m=3$ and 4 multipoles to be misaligned with respect to the ellipsoid. In these models the flux ratio anomalies implied by the standard lens models for PG $1115+080$, B1422+231, and B1933+503 are gone, but the multipole amplitudes are still unreasonably large.

Thus, like Evans \& Witt (2003), we find that adding higher order multipoles to the potential could explain some of the flux ratio anomalies (three of the six in the DK02 sample), if the positions and fluxes of the four compact images were the only available data. The surface density contours implied 

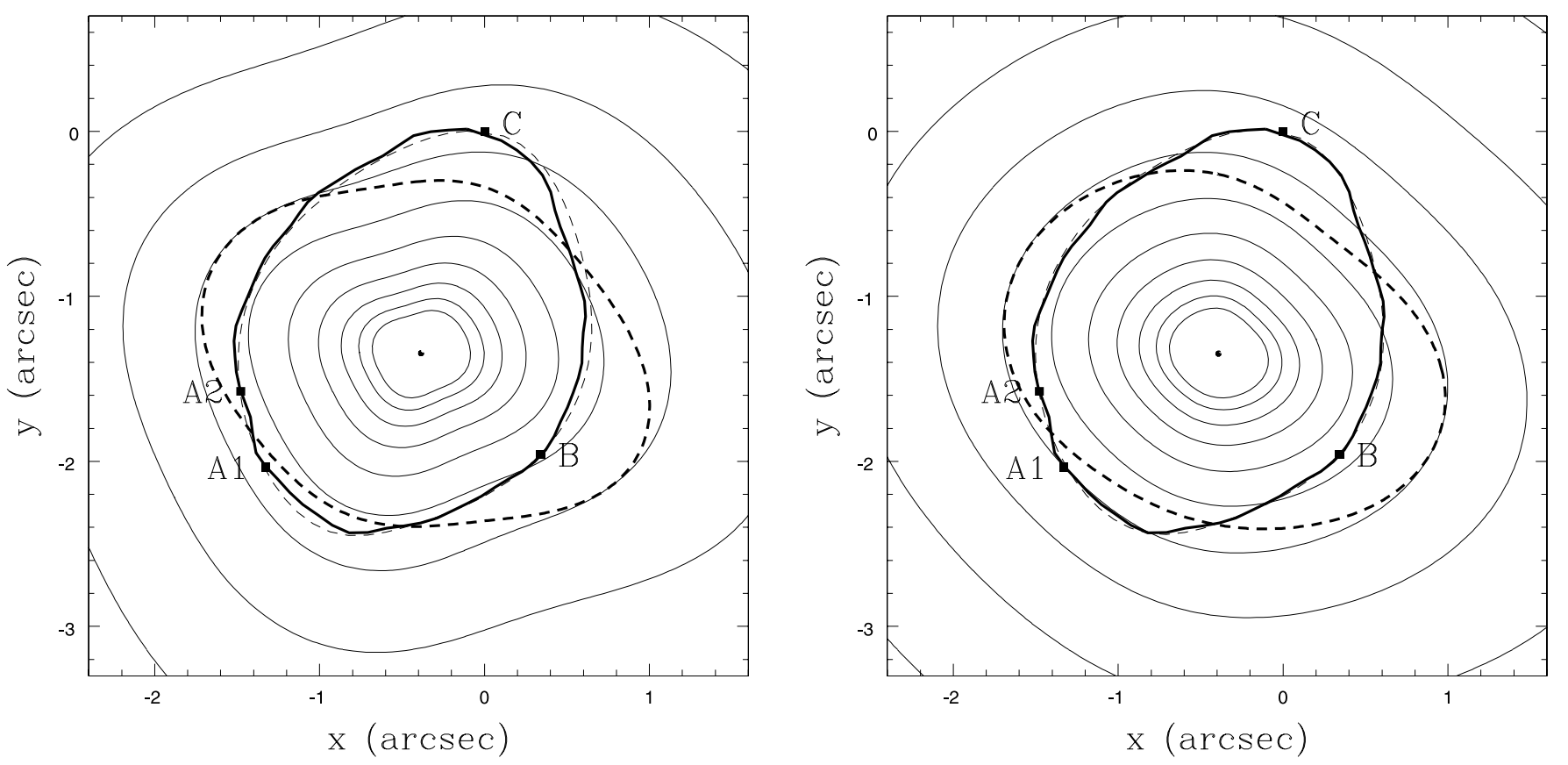

FIG. 4.- Surface density contours for models of PG $1115+080$ including misaligned $a_{3}$ and $a_{4}$ multipoles (thin lines). Left: The model is constrained only by the four compact images $\left(\mathrm{A}_{1}, \mathrm{~A}_{2}, \mathrm{~B}\right.$, and $\mathrm{C}$; squares). Right: The model is also constrained by the Einstein ring image of the host galaxy. The heavy dashed curve is for the tangential critical curve of the lens. The heavy solid curve is for the ring curve of the host galaxy, and the thin dashed curve is for the best-fit model.

by these models are not very physical, as shown for PG $1115+$ 080 and B1933+503 in Figures 4 and 5, respectively. The improved fit with the higher order multipoles is easily understood for $\mathrm{B} 1933+503$. In this lens, image 4 is anomalously bright for an ellipsoidal model, and the new models solve the problem by making the tangential critical line lie much closer to image 4 so that it can be more highly magnified. The cause of the improvement in PG 1115+080, where the anomaly is due to the flux ratio between images $A_{1}$ and $\mathrm{A}_{2}$, is less obvious. In other cases, such as MG 0414+0534, the surface density contours, while very boxy, look more reasonable (Fig. 6).

For three of the DK02 lenses with anomalies, there are additional lensed components beyond the four compact images, which can be used to test these models. In MG 0414+0534 there are VLBI jets associated with each of the quasar images
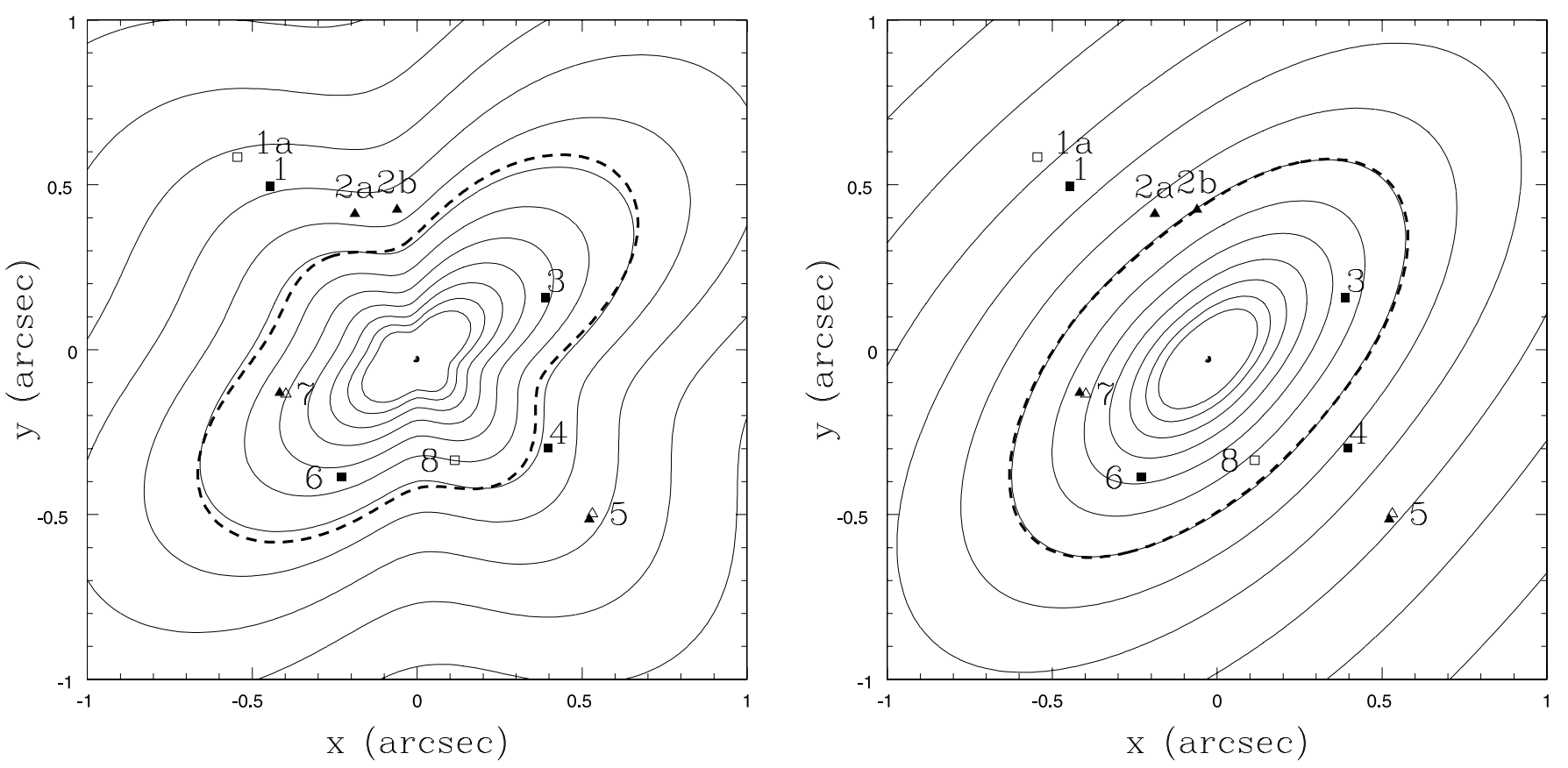

FIG. 5.-Surface density contours for models of B1933+503 including misaligned $a_{3}$ and $a_{4}$ multipoles (thin lines). Left: The model is constrained only by the four compact images (images 1, 3, 4, and 6, filled squares). Right: The model is also constrained by the other images in the lens (the two-image system 1a/8, open squares; the four-image system $2 \mathrm{a} / 2 \mathrm{~b} / 5 / 7$, filled triangles; and the two-image system comprising parts of 5/7, open triangles) The tangential critical line of the model (heavy dashed curve) must pass between the merging images $2 \mathrm{a}$ and $2 \mathrm{~b}$ but fails to do so in the first model (left). 

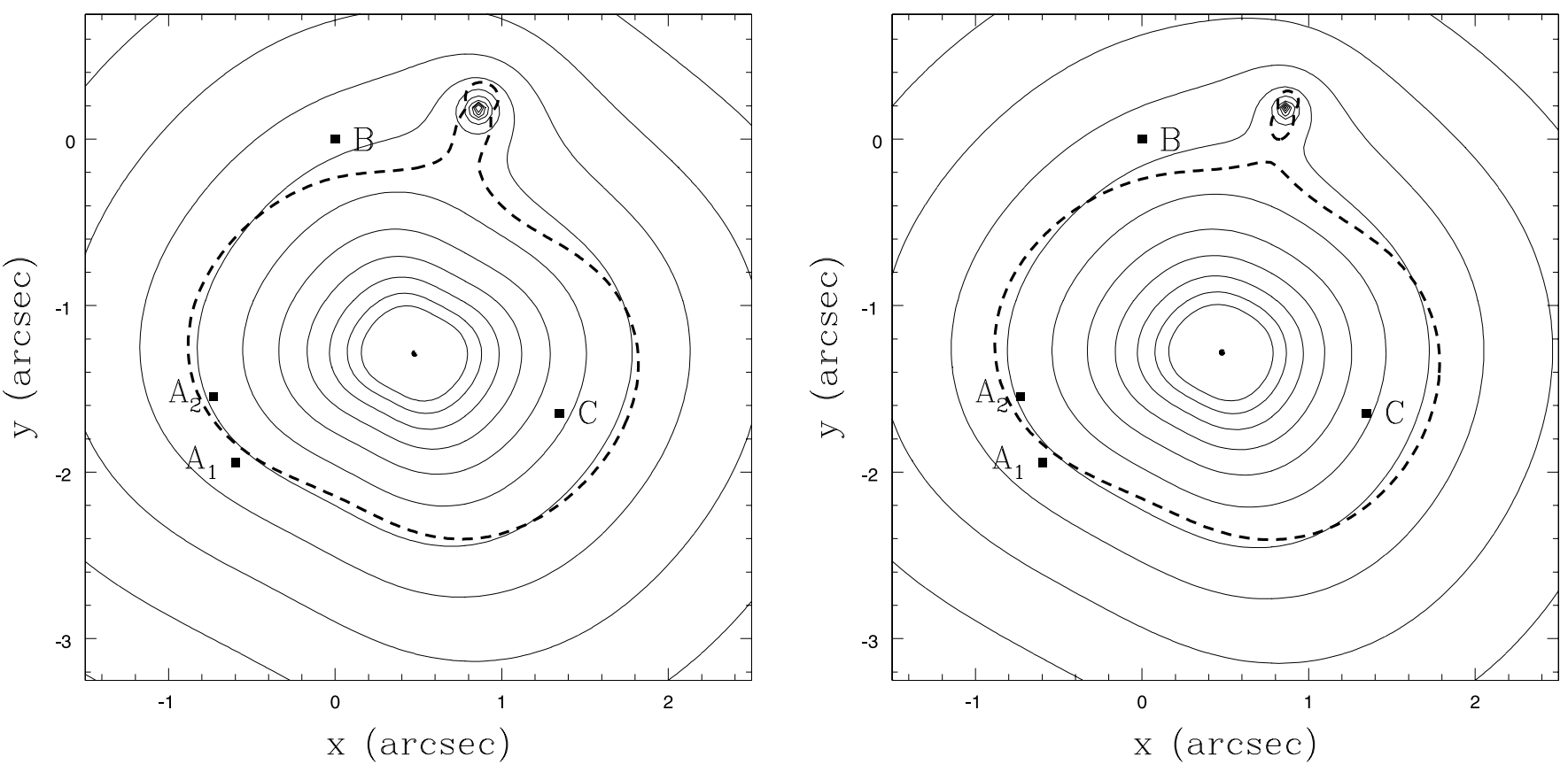

FIG. 6. - Surface density contours for models of MG $0414+0534$ including misaligned $a_{3}$ and $a_{4}$ multipoles (thin lines). Left: The model is constrained only by the four compact images $\left(\mathrm{A}_{1}, \mathrm{~A}_{2}, \mathrm{~B}\right.$, and $\mathrm{C}$, squares). Right: The model is also constrained by the structure of the VLBI jets associated with each image. The heavy dashed curve is for the tangential critical curve of the lens. Note the small satellite galaxy to the north of the lens- this is an example of the high-mass substructures $\left(M \sim 10^{10} M_{\odot}\right)$ that were included as part of the macro model in DK02 instead of being treated as substructure.

(Ros et al. 2000; Trotter et al. 2000), in PG 1115+080 there is the Einstein ring image of the quasar host galaxy (Impey et al. 1998; Kochanek et al. 2001), and in B1933+503 there are additional multiply-imaged components of the radio source (Sykes et al. 1998; Cohn et al. 2001; Muñoz et al. 2001). Table 2 shows the results of fitting the same series of models to these three lenses with the addition of the new constraints. For MG $0414+0534$, where the models were already unable to explain the anomalous flux ratios, little changes with the addition of the VLBI constraints, although the amplitudes of the multipoles change, as shown in Figure 6. For the two lenses where it appeared possible for the higher order multipoles to eliminate the anomalies, we find that the new constraints eliminate these solutions. Figures 4 and 5 show the surface density contours for these new models, and it is immediately clear that the added constraints force the lenses to be significantly more regular and ellipsoidal.

\subsection{Statistical Checks of the Macro Model}

Thus, we find that higher order multipoles can explain only one (B1422+231) of the six anomalous flux ratio lenses in the DK02 sample, given the available data. Keeton et al. (2003) also noted that $\mathrm{B} 1422+231$ was less problematic than many of the other systems. Nonetheless, the perturbation amplitudes needed to explain B1422+231 are much larger than observed in real galaxies, in halo simulations, or in the lenses where additional constraints allow us to determine $a_{3}$ and $a_{4}$. We conclude that higher order multipoles are not a likely explanation, but we would like a more generic test of this conclusion. The basic problem is that most lenses provide only

TABLE 2

Fits Testing the Basic Model

\begin{tabular}{|c|c|c|c|c|c|c|}
\hline Lens & Case & $N_{\text {dof }}$ & $\chi_{\text {tot }}^{2}-\chi_{\mathrm{flx}}^{2}$ & $\chi_{\mathrm{flx}}^{2}$ & $\left|a_{3}\right|$ & $\left|a_{4}\right|$ \\
\hline \multirow[t]{4}{*}{ MG $0414+0534 \ldots \ldots \ldots$} & Astrometry & 20 & 52.7 & 20.8 & $\ldots$ & $\ldots$ \\
\hline & Astrometry+flux & 23 & 53.0 & 19.6 & . & $\ldots$ \\
\hline & $\delta \theta_{m} \equiv 0$ & 21 & 19.5 & 17.6 & 0.000 & 0.032 \\
\hline & Full & 19 & 6.3 & 15.2 & 0.021 & 0.027 \\
\hline \multirow[t]{4}{*}{ PG $1115+080 \ldots \ldots \ldots \ldots$} & Astrometry & 73 & 222.1 & 57.0 & $\ldots$ & $\ldots$ \\
\hline & Astrometry+flux & 76 & 222.7 & 55.6 & $\ldots$ & $\ldots$ \\
\hline & $\delta \theta_{m} \equiv 0$ & 74 & 146.0 & 13.8 & 0.000 & 0.027 \\
\hline & Full & 72 & 121.1 & 22.9 & 0.013 & 0.021 \\
\hline \multirow{4}{*}{$\mathrm{B} 1933+503 \ldots \ldots \ldots \ldots \ldots$} & Astrometry & 11 & 34.4 & 268.2 & $\ldots$ & $\ldots$ \\
\hline & Astrometry+flux & 14 & 33.2 & 256.1 & $\ldots$ & $\ldots$ \\
\hline & $\delta \theta_{m} \equiv 0$ & 12 & 44.7 & 231.2 & 0.007 & 0.001 \\
\hline & Full & 10 & 33.5 & 242.5 & 0.009 & 0.000 \\
\hline
\end{tabular}

Notes.-Model sequences including higher order multipoles for the three lenses with flux ratio anomalies and additional model constraints. The columns are the same as in Table 1. 
enough data to constrain the standard ellipsoidal models, so more complex models that can explain the image fluxes are only constrained by the degree to which the models are viewed as physical.

We noted in $\S 2.2$ that there is a statistical tendency for the anomalous flux ratios to appear as a suppression of the flux of the brightest saddle points relative to the predictions of smooth models and that this was a characteristic property of low optical depth substructure. It is likely that any specific model that can explain the anomalies in individual lenses will be unable to reproduce this statistical property for randomly selected lenses. The worst case for examining this expectation is the effect of the $m=4$ multipole, because it is the largest amplitude higher order multipole with a symmetry matching a common image configuration, cruciform quads like Q2237+ 0305. If an $m=4$ multipole is aligned with the ellipsoid, it will preferentially affect the saddles as compared to the minima of a cruciform quad, but it will symmetrically affect both saddles and both minima. If it is randomly misaligned with respect to the ellipsoid, then it will not distinguish between saddles and minima, and if the observed lens geometries are dominated by merging pairs (fold catastrophes, such as PG $1115+080$ ) or merging triples (cusp catastrophes, such as $\mathrm{B} 1422+231)$, then it will again be unable to distinguish saddles from minima.

We tested these hypotheses for models consisting of an ellipsoid with an aligned (boxy or disky) $a_{4}$ component or a randomly oriented $a_{4}$ component for a range of models for the lens population or typical lens geometry. If we simulate $m=4$ models with the amplitudes typical of real elliptical galaxies or simulated CDM halos $\left(\left|a_{4}\right| \lesssim 0.02\right)$, the resulting $\sim 5 \%$ flux perturbations are too small to be relevant for explaining the anomalous flux ratios. We must use models of abnormally large amplitude, $\left|a_{4}\right|=0.05$, to produce sufficiently large perturbations. After generating an image configuration, we fitted the images using our standard SIE+shear models, fitting only the astrometric data and ignoring the fluxes. We then examined the statistics of the flux residuals after dividing the images into magnification and parity subsamples, just as in $\S 2.2$. We focused on cusp and fold configurations defined by lenses with an opening angle between two images of less than $30^{\circ}$. Of the DK02 sample, the systems MG 0414+0534, $\mathrm{B} 0712+472$, PG $1115+080, \mathrm{~B} 1422+231$, and $\mathrm{B} 2045+265$ would be classified as fold or cusp under this definition, while $\mathrm{B} 1608+656$ and $\mathrm{B} 1933+503$ just fail to make the cut.

The results of our Monte Carlo calculation are displayed in Figures $7 a-7 c$. As expected, $a_{4}$ perturbations to fold or cusp lenses do not systematically distinguish between image parities for disky, boxy, or randomly oriented $m=4$ multipoles. This is in stark contrast to the properties of real fold and cusp lenses, where the bright saddle points are preferentially demagnified compared to the other images (Fig. $7 d$ ). Clearly, $m=4$ perturbations are incapable of producing this behavior, with K-S test probabilities of less than 0.002 that any of these distributions agree with the distribution observed for the cusp/ fold lenses in DK02. We experimented with still higher order multipoles $(m>4)$ and found that they also fare poorly in both systematically demagnifying saddles and differentiating between saddles and minima. The differences between distributions found in the Monte Carlo simulations and in the real data mean that the flux ratio anomalies cannot be due to higher order multipoles. This would be true even if the models with higher order multipoles could successfully model the anomalous flux ratios in every lens!

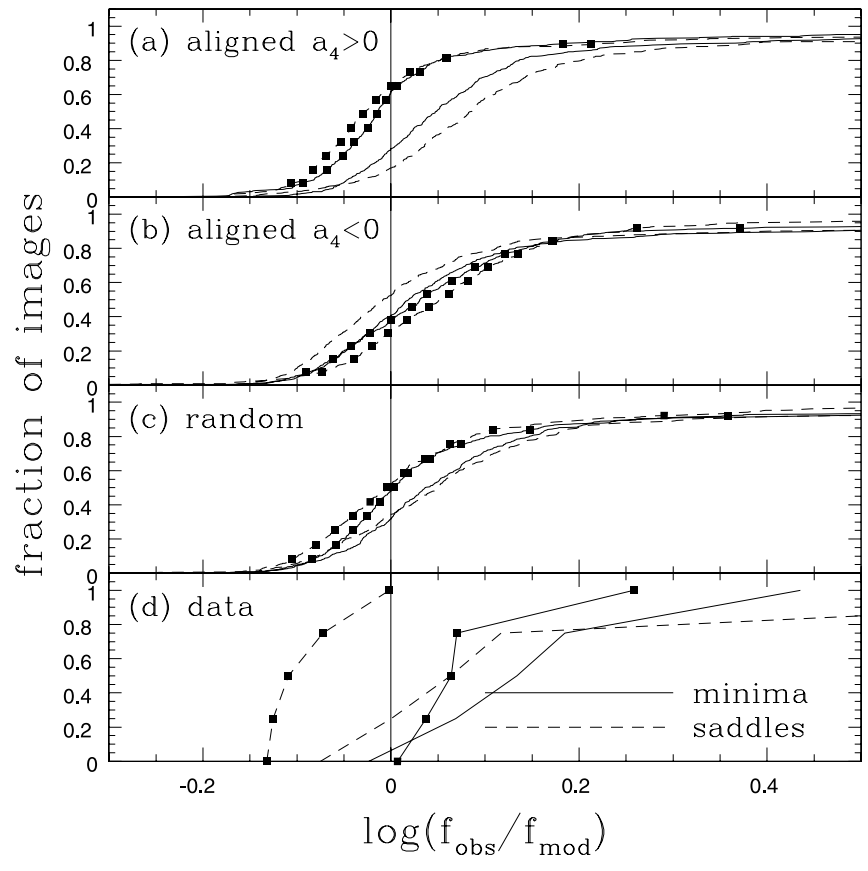

FIG. 7.-Cumulative distributions of model flux residuals, $\log \left(f_{\text {obs }} / f_{\text {mod }}\right)$, expected from Monte Carlo simulations of fold and cusp lenses with $m=4$ components of amplitude $\left|a_{4}\right|=0.05$. $(a, b)$ Perturbations aligned with the major axis of the ellipsoid for $a_{4}>0$ (disky) and $a_{4}<0$ (boxy), respectively. (c) Results for randomly oriented $m=4$ terms. (d) Flux anomalies observed for the five lenses in the DK02 sample satisfying the definition of fold/cusp described in the text. The curve labeling is the same as in Fig. 3.

\section{RULING OUT MICROLENSING OF RADIO SOURCES}

We are left only with substructure in the gravitational potential as a viable explanation of the phenomenon. Real lenses have substructures on two mass scales, stars and satellite halos, so the last step of our argument is to rule out stars as the source of the anomalous flux ratios of lensed radio sources. Microlensing is certainly an important phenomenon in the optical, where its effects are directly observed as uncorrelated time variability in lensed quasars (e.g., Q2237+0305; Woźniak et al. 2000). The principal arguments against significant microlensing in radio lenses are laid out by Koopmans \& de Bruyn (2000). The key point is that the typical scale of the caustic network is set by the Einstein radii of the stars $\left[\theta_{\mathrm{E}}=3\left(M / M_{\odot}\right)^{1 / 2} \mu\right.$ as for a star of mass $M$, a lens at $z_{l}=0.3$, and a source at $z_{s}=2$, hence the name microlensing], while the Compton limit for the minimum possible radio source size is given by

$$
S(\nu)=\frac{2 k T_{b} \nu^{2}}{(1+z) c^{2}} \pi \Delta \theta_{\min }^{2} .
$$

Table 3 shows that the minimum source sizes for the fourimage CLASS lenses are typically $\theta_{\min } \gtrsim 10 \mu \mathrm{as}$, or roughly 10 times larger than $\theta_{\mathrm{E}}$ for a stellar mass function dominated by low-mass stars. A sufficiently nonthermal electron distribution can change these limits, but it is unlikely to reduce them by an order of magnitude. While microlensing leads to fractional flux variations of the order of unity for sources smaller than the typical caustic size, larger sources average over the magnification pattern and show far smaller variations. For sources larger than $20 \mu$ as we would expect $\lesssim 5 \%$ fluctuations from stellar microlensing. For microlensing to produce the $\gtrsim 20 \%$ 
TABLE 3

Compton Limits on CLASS Source Sizes

\begin{tabular}{|c|c|c|c|c|c|}
\hline Lens & $\begin{array}{c}S \\
(\mathrm{mJy})\end{array}$ & $\begin{array}{c}\nu \\
(\mathrm{GHz})\end{array}$ & $z$ & $\mu_{\mathrm{mod}}$ & $\begin{array}{c}\Delta \theta_{\text {min }} \\
(\mu \text { as })\end{array}$ \\
\hline B0128+437................. & 18.9 & 5 & $\ldots$ & 4.3 & 22 \\
\hline MG $0414+0534 \ldots \ldots$ & 149 & 5 & 2.64 & 18 & 33 \\
\hline B0712+472 ............... & 10.5 & 5 & 1.33 & 17 & 7 \\
\hline B1422+231 ............... & 164 & 8.4 & 3.62 & 8.2 & 34 \\
\hline B1555+375 ............... & 17 & 5 & $\ldots$ & 5.2 & 18.6 \\
\hline B1608+656............... & 34.1 & 5 & 1.39 & 5.1 & 23.8 \\
\hline B1933+503 ............... & 17.6 & 8.4 & 2.62 & 3.7 & 14.7 \\
\hline B2045+265 ............... & 29.02 & 1.4 & 1.28 & 52 & 24 \\
\hline
\end{tabular}

NoTES.-Minimum source sizes for radio lenses, based on the brightest image. Sources without redshifts were assumed to have $z=2$. The listed magnifications are from fits to the standard SIE+shear model.

flux anomalies of the radio lenses, we would need to have source sizes of the order of $\sim 1 \mu$ as.

One means of escaping the Compton limit on the radio source sizes is to make most of the radio flux come from a relativistically beamed source. If a significant fraction of the source flux comes from a source moving toward us at relativistic speeds, then the brightness temperature of the beamed component can be increased by a Doppler factor $D=[\gamma(1-$ $\beta \cos i)]^{-1} \sim \gamma$, allowing a corresponding reduction in the source size. For example, there are $\sim 5 \%$ uncorrelated changes in the radio fluxes of the lensed images in B1600+434 that can be explained by microlensing a beamed radio component with $\gamma \gtrsim 10$ (Koopmans \& de Bruyn 2000). Much larger Lorentz factors would be needed for beaming to explain the $\gtrsim 20 \%$ amplitude of the flux anomalies.

In the absence of beaming, microlensing leads to changes in the image flux ratios on timescales of years, while CDM substructure would lead to changes only on timescales of millennia or longer. If there is beaming, so that the characteristic velocities are superluminal rather than simply the peculiar velocities of the lens, source, and observer, then the timescales will be 1000 times shorter. Where radio sources have been monitored for extended periods (e.g., B1600+434: Koopmans \& de Bruyn 2000; B1933+503: Biggs et al. 2000; B1608+656: Fassnacht et al. 2002), the average flux ratios show little time dependence. While this is only weak evidence against microlensing in the absence of beaming, it is strong evidence against microlensing if there is beaming because of the very short timescales created by the relativistic motions. Finally, if microlensing were important, we would expect the flux ratios to have a significant frequency dependence, because we expect the source size to be a function of wavelength. As discussed in $\S 2.1$, this frequency dependence has not been observed.

Because of the stress we have put on the dependence of the flux residuals on image parity, we examined whether the differences between saddle points and minima we discussed above persist for source sizes comparable to the Einstein radii of the substructure. We ran inverse ray-tracing simulations (e.g., Schneider et al. 1992), using $10240^{2}$ rays and the particle mesh (PM) force calculation algorithm (Hockney \& Eastwood 1981). The PM method enjoys several advantages for the microlensing problem, chiefly in the simplicity and speed of the algorithm. Using this code, we simulated microlensing at a saddle point and at a minimum, each with an unperturbed macro-model magnification of about 10 . The

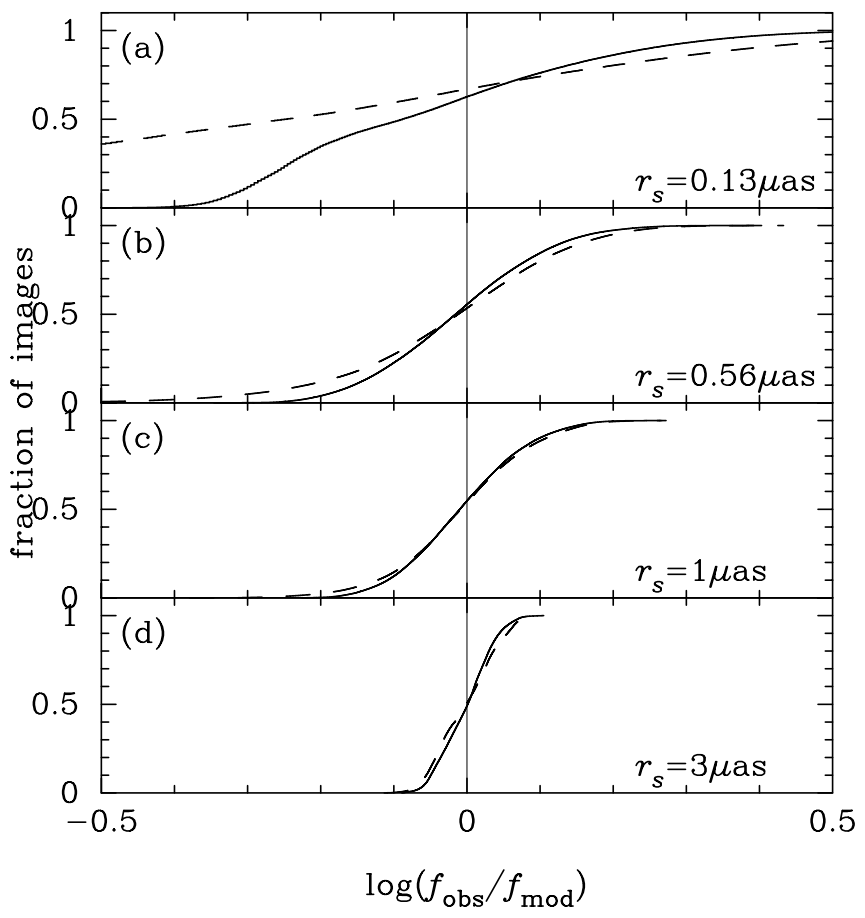

FIG. 8.-Cumulative distributions of magnification perturbations for microlensing, as a function of source size $r_{s}$. The solid lines correspond to a minimum with $\kappa_{0}=0.475$ and $\gamma_{0}=0.425$, while the dashed lines correspond to a saddle point with $\kappa_{0}=0.525$ and $\gamma_{0}=0.575$. The simulations used microlenses with Einstein radii of $\theta_{\mathrm{E}}=1 \mu$ as and surface density $\kappa_{*}=0.075$.

saddle has a total convergence and shear (coming from the macro model) of $\kappa_{0}=0.525, \gamma_{0}=0.575$, while the minimum has $\kappa_{0}=0.475, \gamma_{0}=0.425$. We lay down a random distribution of microlenses with Einstein radii $\theta_{\mathrm{E}}=1 \mu$ as and a number density corresponding to convergence $\kappa_{*}=0.075$. The remaining surface density, $\kappa_{0}-\kappa_{*}$, was in a smooth component.

In Figure 8 we plot the distribution of flux residuals as a function of source size. For a source small compared to $\theta_{\mathrm{E}}$, as in the case corresponding to our grid resolution $\left(r_{s}=\right.$ $0.13 \mu \mathrm{as}$ ), we see that the magnification distribution for the saddle point is skewed toward demagnification compared to that for the minimum, in agreement with previous results (e.g., Schechter \& Wambsganss 2002; see their Fig. 3). For larger sources, which we illustrate for the cases $r_{s}=\theta_{\mathrm{E}}=1 \mu$ as and $3 \theta_{\mathrm{E}}=3 \mu \mathrm{as}$, the widths of the distribution narrow rapidly (with $\sigma_{\mu} / \mu_{0} \propto 1 / r_{s}$, roughly; e.g., Koopmans \& de Bruyn 2000), and the differences between the distributions for saddle points and minima vanish. The mean $\left\langle\log \mu / \mu_{0}\right\rangle$ provides a measure of the skewness, and for source sizes of $r_{s}=0.13$, $0.56,1$, and $3 \mu$ as we find values of $\left\langle\log _{10} \mu / \mu_{0}\right\rangle=-0.26$, $-0.025,-0.01$, and -0.002 , respectively. Evidently, even source sizes as small as $\theta_{\mathrm{E}} / 2=0.5 \mu$ as are sufficient to erase the asymmetry in the distribution of perturbations to saddle point images. In reality, radio QSOs are expected to have much larger angular sizes (10-30 $\mu$ as; see Table 3$)$, giving even narrower, more symmetric distributions than those plotted in Figure 8.

\section{SUMMARY AND DISCUSSION}

We have considered three alternatives to substructure as the source of the flux anomalies observed in four-image radio lenses: propagation effects, such as refractive scattering in the 
interstellar medium (ISM) of the lens galaxy, problems in the macro model describing the gravitational potential of the lens galaxy, and microlensing by ordinary stars in the lens galaxy. We find that none of these alternatives is likely to explain the phenomenon.

Generating the flux anomalies using the ISM fails on two counts. First, the anomalies in the flux ratios of the radio lenses are essentially independent of wavelength, unlike any normal propagation effect in the ISM (e.g., scintillation, refractive scattering, and free-free absorption). Any attempt to use the ISM as an explanation of the anomalous flux ratios must find a mechanism to produce a nearly frequency-independent optical depth - the radio equivalent of the "gray dust" proposals for Type Ia supernovae (Aguirre 1999). Second, the flux ratio anomalies depend on the image parity and magnification. In particular, the brightest saddle point image has a tendency to be demagnified relative to the other images, which is a characteristic of low optical depth substructure (Schechter \& Wambsganss 2002; Keeton 2003). Since the ISM should preferentially perturb the least magnified images, which will be the most compact, and show no dependence on the image parity, we can statistically rule out any propagation effect.

Solving the flux anomalies by modifying the smooth gravitational potential of the lens also fails on two counts. Following other studies (Evans \& Witt 2003; Möller et al. 2003; Quadri et al. 2003), we investigated adding higher order multipoles $(\phi \propto \cos m \theta$, with $m>2)$ to the potential. For the models we attempted (which were restricted to adding $m=3$ and 4), we could find solutions without flux anomalies for only one of the six lenses from DK02 that had anomalies in our standard ellipsoidal lens models. The fits required the higher order multipoles to be significantly misaligned with respect to the axes of the ellipsoid and had perturbation amplitudes larger than seen in real galaxies or halo simulations. In three of the lenses, the presence of additional constraints, either Einstein ring images of the quasar host galaxy or additional lensed components of the radio source, allowed us to measure directly the magnitude of the higher multipoles in the gravitational potential. In every instance, the $m=3$ and 4 multipoles were constrained to the small amplitudes typically observed in galaxies and halo models, largely justifying the use of ellipsoidal mass models to describe lens galaxies.

The second problem with the higher order multipole models is that they generally cannot reproduce the observed parity dependence of the flux anomalies. They can do so in very specific cases where the symmetry of the lens (a cruciform quad like Q2237+0305) matches the symmetry of the multipole $[a \cos (4 \theta)$ multipole] and the multipole has very large amplitude and is aligned with the dominant ellipsoidal potential. However, none of the radio quads with anomalies have this symmetry (they generally have merging image pairs or triples associated with fold and cusp caustics), and the models require multipoles that are not aligned with the ellipsoid. If we examine the distribution of anomalies found by fitting lenses with higher order multipoles using only standard ellipsoidal lens models, we find that the statistical properties of the brightest saddle point do not stand out from those of the other images. This allows us to rule out these models as an explanation even if they could fit the anomalies in each individual lens (which they cannot).

The failure of the ISM or changes in the smooth potential to explain the data means that the explanation must be substructure in the potential. These substructures must be either dark objects, since luminous satellites are not abundant enough to account for the preponderance of anomalous fluxes (e.g., Chiba 2002), or the stellar populations in the lens galaxy. Either source of substructure will lead to the statistical differences between saddle points and minima if the optical depth is low (Schechter \& Wambsganss 2002; Keeton 2003). The angular scales of the magnification patterns are very different for stars (microarcseconds) and satellites (milliarcseconds), and by using radio sources we should be averaging out the stellar magnification patterns, to leave only the contribution from the satellites. We tested this by taking typical microlensing patterns for saddle point and minimum images, which show the expected differences for point sources, and smoothing on larger and larger scales. As expected, the magnification distributions for the saddle point and the minimum show significant differences only when the source size is smaller than the Einstein radius of the stars. Source sizes of even 1 mas are sufficient to eliminate the differences, so radio sources could have angular sizes even an order of magnitude smaller than the Compton limit without microlensing making a significant contribution to the flux anomalies of radio lenses.

In summary, CDM substructure remains the best explanation of the flux ratio anomalies of gravitational lenses. The most powerful piece of evidence is the statistically significant differences between the anomalies in saddle points and minima and between the bright and faint images. Substructure makes a very specific prediction that the brightest saddle point should show a distribution that is very different from that of the other images, as observed in the data, and is very difficult or impossible to reproduce using either the ISM or changes in the smooth potential.

However, the issues raised by these considerations point to future observations that can further clarify the origin of anomalous fluxes in lens systems. ISM effects can be more strongly constrained by measuring flux ratios at still higher frequencies (e.g., $43 \mathrm{GHz}$ at the VLA). Mid-infrared $(5-10 \mu \mathrm{m})$ flux ratios, where the wavelength is far too short to be bothered by electrons and far too long to be bothered by dust, are difficult to measure but completely insensitive to the ISM (e.g., Agol et al. 2000). The mid-infrared source sizes should also be large enough to be affected only by satellites, adding a further check for whether we can be misinterpreting microlensing effects as substructure. Integral field spectroscopy, to compare the flux ratios in the optical continuum to those in the broad emission lines, provides another way to separate the effects of microlensing and substructure, because the broad line-emitting regions should be significantly larger than the source of the optical continuum (e.g., Moustakas \& Metcalf 2003). Monitoring the lenses, in either the radio or the optical, to look for changes in the flux ratios can also be used to distinguish microlensing effects from CDM substructure. Observations to find additional lensed structures are the best approach to determining whether more complicated lens potentials are needed. Clean constraints on more complicated angular structures can be obtained by analyzing the shapes of the Einstein ring images of host galaxies found in deep, high-resolution, infrared images (see Kochanek et al. 2001).

We thank Roger Blandford, Scott Gaudi, Gil Holder, Chuck Keeton, Feryal Özel, and David Rusin for helpful discussions. We also thank Chuck Keeton for rapidly adding the higher 
order multipole potentials to the lensmodel package and Chris Fassnacht for giving us the unpublished 5 and $15 \mathrm{GHz}$ flux measurements for B1608+656. N. D. acknowledges the support of NASA through Hubble Fellowship grant HST-HF-
01148.01-A, awarded by STScI, which is operated by AURA, Inc. for NASA, under contract NAS5-26555. C. S. K. is supported by the Smithsonian Institution, NASA grant NAG5-9265, and STScI grant GO-9375.
Agol, E., Jones, B., \& Blaes, O. 2000, ApJ, 545, 657

Aguirre, A. N. 1999, ApJ, 512, L19

Bade, N., Siebert, J., Lopez, S., Voges, W., \& Reimers, D. 1997, A\&A, 317, L13

Bender, R., Surma, P., Döbereiner, S., Möllenhoff, C., \& Madejsky, R. 1989, A\&A, 217, 35

Biggs, A. D., Xanthopoulos, E., Browne, I. W. A., Koopmans, L. V. E., \& Fassnacht, C. D. 2000, MNRAS, 318, 73

Bode, P., Ostriker, J. P., \& Turok, N. 2001, ApJ, 556, 93

Bradac, M., Schneider, P., Steinmetz, M., Lombardi, M., King, L. J., \& Porcas, R. 2002, A\&A, 388, 373

Burkert, A., \& Naab, T. 2003, in Galaxies and Chaos, ed. G. Contopoulos \& N. Voglis (Berlin: Springer), 327

Chiba, M. 2002, ApJ, 565, 17

Cohn, J. D., Kochanek, C. S., McLeod, B. A., \& Keeton, C. R. 2001, ApJ, 554,1216

Dalal, N., \& Kochanek, C. S. 2002, ApJ, 572, 25 (DK02)

Evans, N. W., \& Witt, H. J. 2001, MNRAS, 327, 1260 2003, MNRAS, 345, 1351

Falco, E. E., et al. 1999, ApJ, 523, 617

Fassnacht, C. D., Womble, D. S., Neugebauer, G., Browne, I. W. A., Readhead, A. C. S., Matthews, K., \& Pearson, T. J. 1996, ApJ, 460, L103

Fassnacht, C. D., Xanthopoulos, E., Koopmans, L. V. E., \& Rusin, D. 2002, ApJ, 581, 823

Fassnacht, C. D., et al. 1999, AJ, 117, 658

Hewitt, J. N., Turner, E. L., Lawrence, C. R., Schneider, D. P., \& Brody, J. P. 1992, AJ, 104, 968

Heyl, J. S., Hernquist, L., \& Spergel, D. N. 1994, ApJ, 427, 165

Hockney, R. W., \& Eastwood, J. W. 1981, Computer Simulation Using Particles (New York: McGraw-Hill)

Huchra, J., Gorenstein, M., Kent, S., Shapiro, I., Smith, G., Horine, E., \& Perley, R. 1985, AJ, 90, 691

Impey, C. D., Falco, E. E., Kochanek, C. S., Lehár, J., McLeod, B. A., Rix, H.-W., Peng, C. Y., \& Keeton, C. R. 1998, ApJ, 509, 551

Inada, N., et al. 2003, AJ, 126, 666

Jackson, N., et al. 1998, MNRAS, 296, 483

Katz, C. A., Moore, C. B., \& Hewitt, J. N. 1997, ApJ, 475, 512

Kauffmann, G., White, S. D. M., \& Guiderdoni, B. 1993, MNRAS, 264, 201

Keeton, C. R. 2001a, ApJL, submitted (astro-ph/0111595)

. 2001b, preprint (astro-ph/0102341)

2003, ApJ, 584, 664

Keeton, C. R., Gaudi, B. S., \& Petters, A. O. 2003, ApJ, 598, 138

Klypin, A., Kravtsov, A. V., Valenzuela, O., \& Prada, F. 1999, ApJ, 522, 82

Kochanek, C. S. 2002, in The Shapes of Galaxies and Their Dark Halos, ed

P. Natarajan (Singapore: World Scientific), 62

\section{REFERENCES}

Kochanek, C. S., Keeton, C. R., \& McLeod, B. A. 2001, ApJ, 547, 50

Koopmans, L. V. E., \& de Bruyn, A. G. 2000, A\&A, 358, 793

Magain, P., Surdej, J., Swings, J.-P., Borgeest, U., Kayser, R., Kühr, H., Refsdahl, S., \& Remy, M. 1988, Nature, 334, 325

Mao, S., \& Schneider, P. 1998, MNRAS, 295, 587

Marlow, D. R., Browne, I. W. A., Jackson, N., \& Wilkinson, P. N. 1999a, MNRAS, 305, 15

Marlow, D. R., et al. 1999b, AJ, 118, 654

Metcalf, R. B. 2002, ApJ, 580, 696

Metcalf, R. B., \& Madau, P. 2001, ApJ, 563, 9

Metcalf, R. B., \& Zhao, H. 2002, ApJ, 567, L5

Mezger, P. C., \& Henderson, A. P. 1967, ApJ, 147, 471

Möller, O., Hewett, P., \& Blain, A. W. 2003, MNRAS, 345, 1

Moore, B. Ghigna, S., Governato, F., Lake, G., Quinn, T., Stadel, J., \& Tozzi, P. 1999, ApJ, 524, L19

Moustakas, L. A., \& Metcalf, R. B. 2003, MNRAS, 339, 607

Muñoz, J. A., Kochanek, C. S., \& Keeton, C. R. 2001, ApJ, 558, 657

Myers, S. T., et al. 1999, AJ, 117, 2565

Narayan, R. 1992, Philos. Trans. Phys. Sci. Eng., 341, 151

Patnaik, A. R., Browne, I. W. A., Walsh, D., Chaffee, R. H., \& Foltz, C. B. 1992, MNRAS, 259, 1P

Patnaik, A. R., \& Narasimha, D. 2001, MNRAS, 326, 1403

Phillips, P. M., et al. 2000, MNRAS, 319, L7

Quadri, R., Möller, O., \& Natarajan, P. 2003, ApJ, 597, 659

Reimers, D., Hagen, H.-J., Baade, R., Lopez, S., \& Tytler, D. 2002, A\&A, 382, L26

Rest, A., van den Bosch, F. C., Jaffe, W., Tran, H., Tsvetanov, Z., Ford, H. C., Davies, J., \& Schafer, J. L. 2001, AJ, 121, 2431

Ros, E., Guirado, J. C., Marcaide, J. M., Pérez-Torres, M. A., Falco, E. E., Muñoz, J. A., Alberdi, A., \& Lara L. 2000, A\&A, 362, 845

Schechter, P. L., \& Wambsganss, J. 2002, ApJ, 580, 685

Schneider, P., Ehlers, J., \& Falco, E. E. 1992, Gravitational Lenses (Berlin: Springer)

Sykes, C. M., et al. 1998, MNRAS, 301, 310

Trotter, C. S., Winn, J. N., \& Hewitt, J. N. 2000, ApJ, 535, 671

Weymann, R. J., et al. 1980, Nature, 285, 641

Wisotzki, L., Christlieb, N., Liu, M. C., Maza, J., Morgan, N. D., \& Schechter, P. L. 1999, A\&A, 348, L41

Wisotzki, L., Schechter, P. L., Bradt, H. V., Heinmüller, J., \& Reimers, D. 2002, A\&A, 395, 17

Woźniak, P. R., Udalski, A., Szymański, M., Kubiak, M., Pietrzyński, G., Soszyński, I., \& Żebruń, K. 2000, ApJ, 540, L65

Zentner, A. R., \& Bullock, J. S. 2003, ApJ, 598, 49 